\title{
Case Report \\ Fatal Fulminant Hepatic Failure in a Diabetic with Primary Dengue
}

\section{Stalin Viswanathan, Nayyar Iqbal, P. Philip Anemon, and G. Shyam Kumar}

Department of Medicine, Pondicherry Institute of Medical Sciences, Kalapet, Pondicherry 605014, India

Correspondence should be addressed to Stalin Viswanathan, stalinviswanathan@ymail.com

Received 30 September 2010; Accepted 13 December 2010

Academic Editor: Ib Christian Bygbjerg

Copyright (๑) 2010 Stalin Viswanathan et al. This is an open access article distributed under the Creative Commons Attribution License, which permits unrestricted use, distribution, and reproduction in any medium, provided the original work is properly cited.

We report a 49-year-old diabetic with dengue hemorrhagic fever who developed fulminant hepatitis, severe coagulopathy, shock, and refractory metabolic acidosis and died on the eighth day of illness. This is the only second report of an adult with fatal fulminant hepatic failure due to dengue, and the first case arising from a primary dengue infection.

\section{Introduction}

Dengue is an arboviral illness caused by four serotypes (DEN-V 1 to 4 ) and is transmitted by the bite of an infected Aedes mosquito with an incubation period varying from 3 to 14 days [1]. Hepatic failure in dengue has been frequently reported in the pediatric population and is rare in adults [2]. Fulminant hepatic failure has been reported only in six adults as of 2007, one of whom, a Bangladeshi immigrant to England, had had a fatal illness with secondary dengue [3]. We report a case of fulminant hepatic failure arising from a primary dengue infection in a middle-aged diabetic that ended in his death.

\section{Case Report}

This 49-year-old bus driver was admitted with complaints of fever, headache, bodyaches, nausea, and fatigue for 5 days prior to admission. He had been on oral hypoglycemic agents for the previous three years and did not smoke and was a teetotaler. Examination revealed a temperature of $100^{\circ} \mathrm{F}$, tachycardia (105/min), normotension, and tachypnea (28/minute) in a tired and drowsy looking obese man (BMI$\left.32 \mathrm{~kg} / \mathrm{m}^{2}\right)$. He had a palpable liver of $5 \mathrm{~cm}$ and rightsided pleural effusion (Figure 1). Pending reports, he was instituted on intravenous ceftriaxone and artesunate and oral doxycycline. Investigations showed hemoconcentration, thrombocytopenia, direct hyperbilirubinemia, transaminases elevation (aspartate transaminase/alanine transaminase $($ AST/ALT $)=2.3$ ), prolonged activated partial thromboplastin time (aPTT) and prothrombin time, and prerenal azotemia with hyperkalemia (Table 1). Dengue IgM and NS1 antigen were positive, while malarial smear and scrub IgM ELISA were negative. Hepatitis panel and HIV were negative. Serum ceruloplasmin was normal. An abdominal ultrasonogram (day 2) revealed hepatomegaly, gall bladder edema, moderate ascites, and bilateral pleural effusion.

An internal jugular central venous access was secured following platelet transfusion, and intravenous fluids (dextrose saline), fresh frozen plasma (FFP), intravenous vitamin $\mathrm{K}$ and thiamine, lactulose, and oral metronidazole were administered. Six hours after admission, he was intubated and mechanically ventilated for worsening sensorium and respiratory distress. Eleven hours after admission he developed shock, despite normal-to-high central venous pressures and metabolic acidosis poorly responsive to noradrenaline, dopamine, and bicarbonate infusions. His transaminase levels continued to increase (AST $>$ ALT $=5$; Table 1 ), and coagulation parameters could not be corrected despite transfusion of packed cells (3 units) and FFP (14 units) over 42 hours of hospital stay which ended fatally for this man with dengue hemorrhagic fever. 


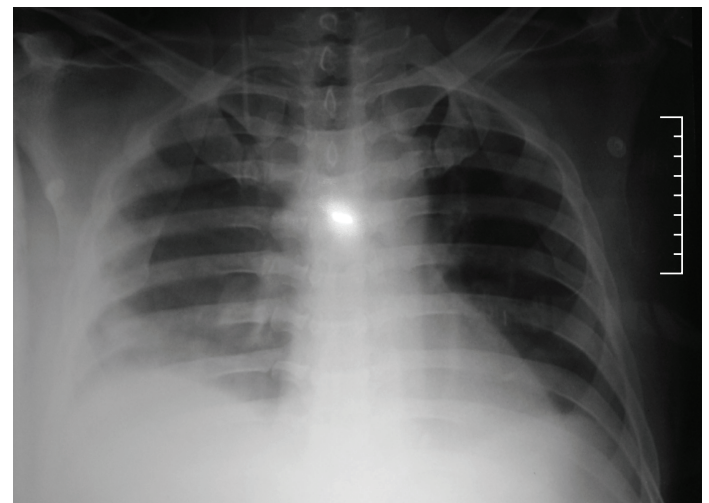

FIGURE 1: Chest radiograph on day 1 with right-sided pleural effusion.

TABLE 1: Laboratory values.

\begin{tabular}{lccc}
\hline Parameter & Day 1 & Day2 & Day 3 \\
\hline Hemoglobin g/dL & 18.9 & 14.3 & 8.6 \\
Hematocrit & 51.8 & 42 & 27 \\
Platelets/cumm & 15000 & 9000 & 21000 \\
Leukocyte/cumm & 10700 & & 20400 \\
Urea (mg/dL) & 49 & 59 & 63 \\
Creatinine (mg/dL) & 1.6 & 2.8 & 4.7 \\
Total bilirubin (mg) & 5.7 & 4.6 & \\
Direct bilirubin (mg) & 3.1 & 3 & \\
Aspartate transaminase U/L & 8980 & & 28050 \\
Alanine transaminase U/L & 3850 & & 5580 \\
Alkaline phopshatase U/L & 206 & & 290 \\
Total protein g/dL & 6.4 & & 5 \\
Serum albumin g/dL & 3.8 & & 2.9 \\
Creatine kinase U/L & 46 & & \\
aPTT prolongation (seconds) & 46 & 75 & 47 \\
Prothrombin time prolongation (seconds) & 31 & 52 & 39 \\
\hline
\end{tabular}

\section{Discussion}

Dengue hemorrhagic fever (DHF) is defined by the presence of fever, thrombocytopenia, hemorrhagic tendencies, and evidence of capillary leak [4]. Dengue hemorrhagic fever generally occurs in secondary infection of another serotype and is more common in children, but studies in Thailand have shown an increasing prevalence in adults; moreover, an association with secondary infection seems less likely in adults [4]. Case fatalities are highest in children, and in developing countries DHF has a mortality of 5\% [5]. Studies in travelers, naïve to the dengue virus, on returning from endemic regions have shown a similar mortality in DHF to that of people residing in endemic areas [4].

In DHF, an antibody-dependent immune mechanism is in play, by which nonneutralizing antibodies of previous dengue infection complexes with the new infecting serotype enhancing its uptake [1]. Liver damage is due to viral replication in hepatocytes, cytokine-related damage, and partly due to ischemic hepatitis $[6,7]$, but in our patient, with worsening hemodynamic parameters and necessity of inotropic support, the bilirubin remained stable and AST/ALT ratio did not reverse (Table 1). Also a dengue virus-specific CD4+ and CD8+ T cells may cause cytolysis [1]. The dengue virus targets both the hepatocyte as well as kupffer cell and following internalization induces tumor necrosis factor-related apoptotic damage [8]. The virus enters the hepatocytes and kupffer cells by phagocytosis and receptor-mediated endocytosis, respectively, [1]. Dengue characteristically causes zone 2 necrosis of the liver, and the changes are similar to those of the yellow fever [8]. Liver biopsy usually reveals massive infiltration by the virus, with minimal mononuclear cell infiltrate [9]. Other findings include Councilman bodies, kupffer cell hyperplasia, and steatosis [1].

Dengue can cause mild elevations in transaminases, but the levels seen in our patient have not been previously reported. Transaminases can be elevated five to ten times the normal in DHF [6]. Acute hepatitis is more common in dengue fever, while fulminant hepatic failure is more common in DHF $[6,9]$. Acute hepatitis is seen in less than four percent of patients with dengue [10]. Aspartate transaminase [AST] elevation is more commoner and higher than ALT elevations, and this is similar to that of alcoholic hepatitis $[1,8]$. Transaminase elevations peak on the 7 th to 8th day [6]. ALT elevation at par with AST levels generally reflects hepatic cell necrosis. Our patient had AST/ALT of $8980 / 3850 \mathrm{U} / \mathrm{L}$ at admission which increased to 28050/5580 U/L on day 3 of hospitalization (day 8 of illness). He also had thrombocytopenia of $15000 / \mathrm{mm}^{3}$ that fell to $9000 / \mathrm{mm}^{3}$ on day 2 without overt bleeding manifestations.

Jaundice and raised alkaline phosphatase have also been observed in DHF as was seen in our patient [7]. Factors which worsen liver disease in dengue include presence of shock, acidosis, and preexisting alcoholic liver disease, diabetes, hepatotoxic drug use, sickle cell disease, and race [6, 7]. Coexisting Hepatitis B infection does not seem to worsen the prognosis although acetaminophen and antiemetic usage may predilect to further liver damage [1]. Shock, acidosis, severe thrombocytopenia, bleeding diathesis, and encephalopathy were associated with mortality in dengue $[6,10]$. Dengue encephalopathy is associated with very high levels of transaminases [8]. Our patient developed shock requiring inotropes and had refractory metabolic acidosis; he was a teetotaler but was an obese diabetic and might have had a component of nonalcoholic fatty liver disease. Serotypes 1 and 3 are more hepatotropic than the others although all four serotypes can be associated with hepatic dysfunction [6]. Also some serotypes may have more epidemic potential than the others, and therefore they replicate faster leading to higher levels of viremia [1]. We did not have facility for serotype identification in our hospital. In view of shock, myocarditis and pancreatitis were ruled out by normal levels of cardiac enzymes, amylase, lipase, and a bedside echocardiography.

In conclusion, primary dengue can rarely cause fulminant hepatic failure and death, which may be prevented by early and aggressive supportive measures that include volume resuscitation, treatment of coagulopathy, acidosis, and encephalopathy. 


\section{References}

[1] S. L. Seneviratne, G. N. Malavige, and H. J. de Silva, "Pathogenesis of liver involvement during dengue viral infections," Transactions of the Royal Society of Tropical Medicine and Hygiene, vol. 100, no. 7, pp. 608-614, 2006.

[2] S. Giri, M. P. Agarwal, V. Sharma, and A. Singh, "Acute hepatic failure due to dengue: a case report," Cases Journal, vol. 1, p. 204, 2008.

[3] V. Subramanian, S. Shenoy, and A. J. Joseph, "Dengue hemorrhagic fever and fulminant hepatic failure," Digestive Diseases and Sciences, vol. 50, no. 6, pp. 1146-1147, 2005.

[4] E. Meltzer and E. Schwartz, "A travel medicine view of dengue and dengue hemorrhagic fever," Travel Medicine and Infectious Disease, vol. 7, no. 5, pp. 278-283, 2009.

[5] S. R. Kamath and S. Ranjit, "Clinical features, complications and atypical manifestations of children with severe forms of dengue hemorrhagic fever in South India," Indian journal of pediatrics, vol. 73, no. 10, pp. 889-895, 2006.

[6] L. M. Ling, A. Wilder-Smith, and Y. S. Leo, "Fulminant hepatitis in dengue haemorrhagic fever," Journal of Clinical Virology, vol. 38, no. 3, pp. 265-268, 2007.

[7] S. Gulati and A. Maheshwari, "Atypical manifestations of dengue," Tropical Medicine and International Health, vol. 12, no. 9, pp. 1087-1095, 2007.

[8] J. Gasperino, J. Yunen, A. Guh, K. E. Tanaka, V. Kvetan, and H. Doyle, "Fulminant liver failure secondary to haemorrhagic dengue in an international traveller," Liver International, vol. 27, no. 8, pp. 1148-1151, 2007.

[9] F. Carvalho De MacEdo, A. F. Nicol, L. D. Cooper, M. Yearsley, A. R. Cordovil Pires, and G. J. Nuovo, "Histologic, viral, and molecular correlates of dengue fever infection of the liver using highly sensitive immunohistochemistry," Diagnostic Molecular Pathology, vol. 15, no. 4, pp. 223-228, 2006.

[10] I. Shah, "Dengue and liver disease," Scandinavian Journal of Infectious Diseases, vol. 40, no. 11-12, pp. 993-994, 2008. 


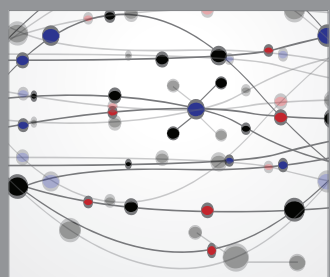

The Scientific World Journal
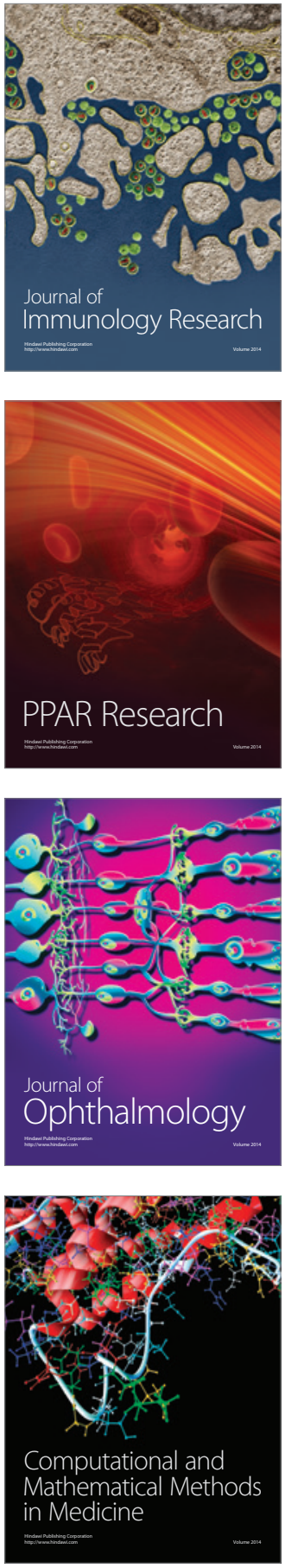

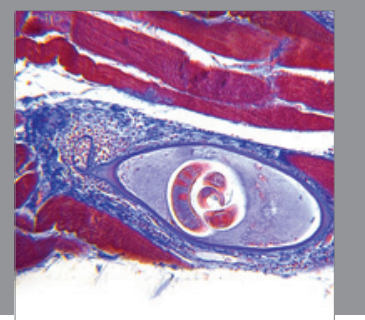

Gastroenterology

Research and Practice
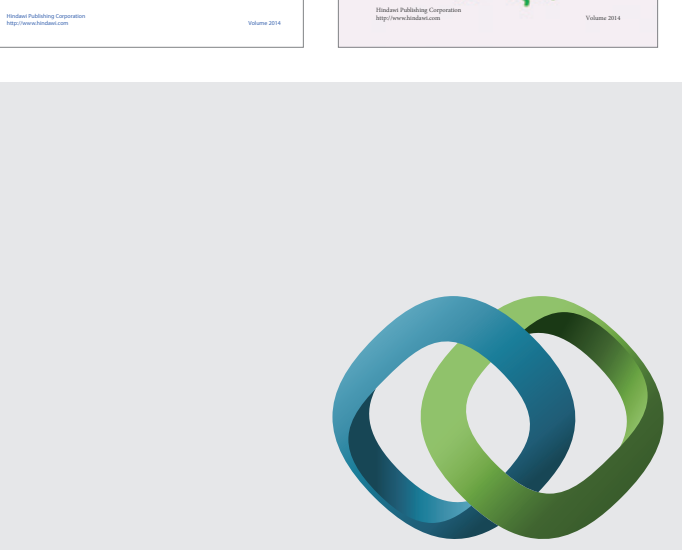

\section{Hindawi}

Submit your manuscripts at

http://www.hindawi.com
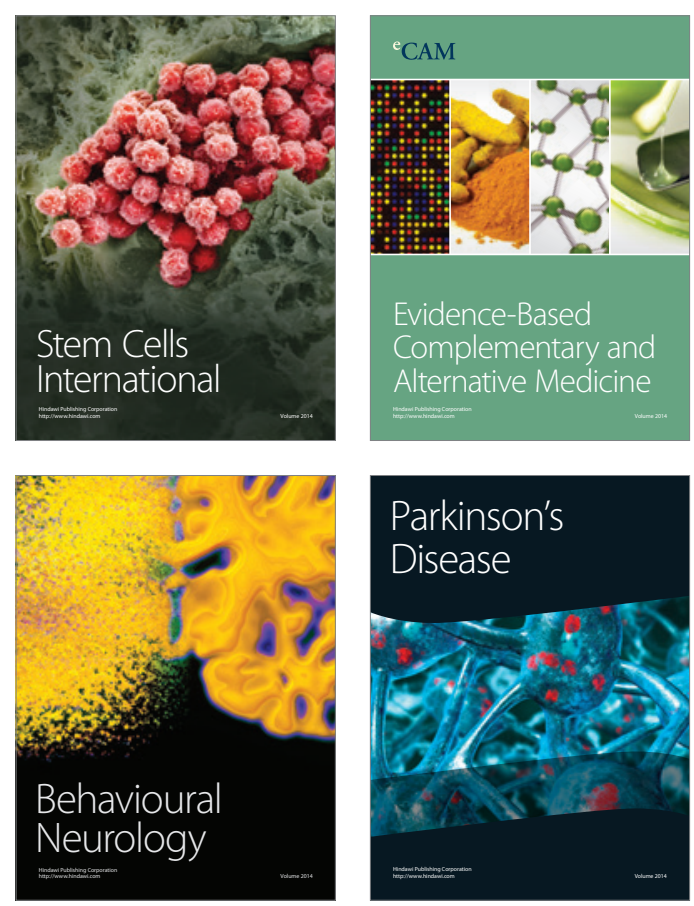

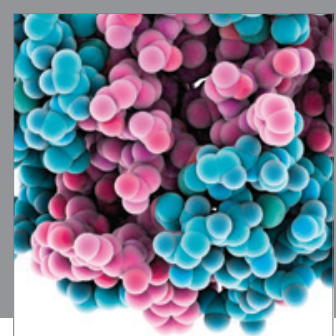

Journal of
Diabetes Research

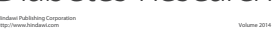

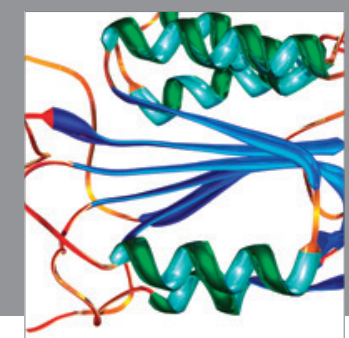

Disease Markers
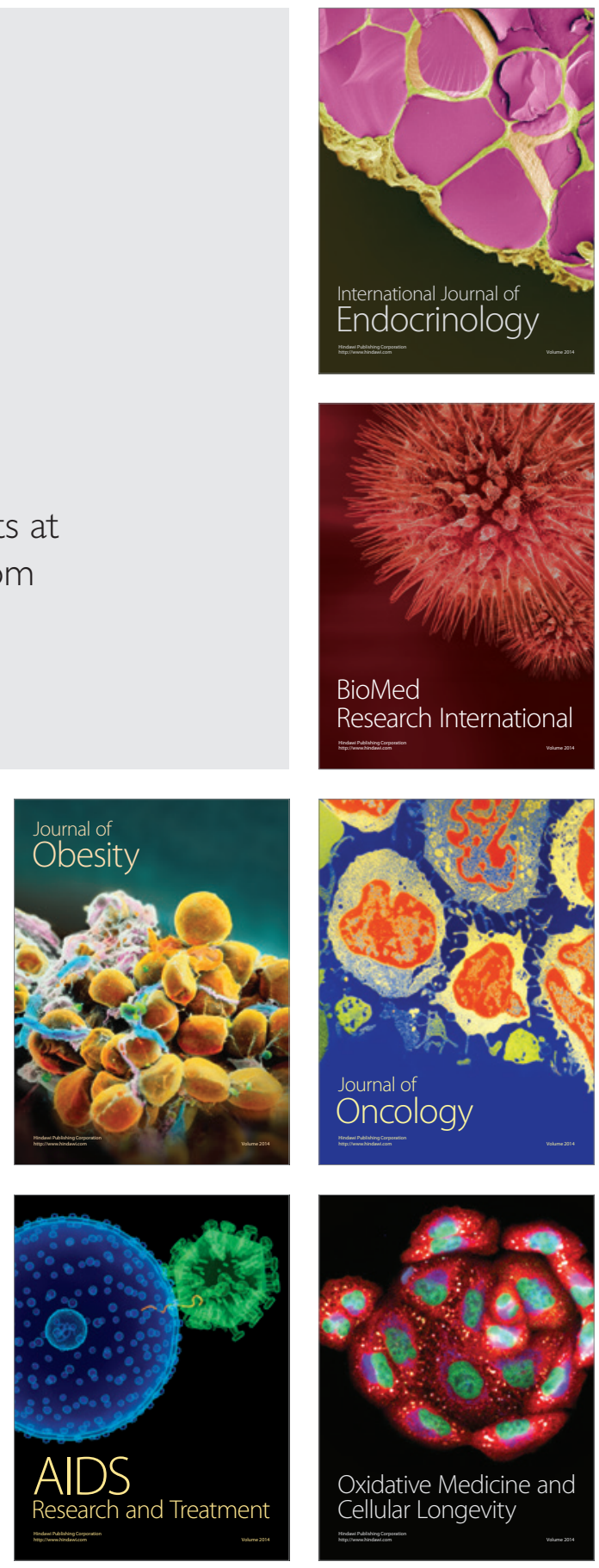